\title{
Extraskeletal Mesenchymal Chondrosarcoma, a Rare Entity with Unusual Metastases: A Case Report
}

\author{
Afaque Ali, Sara Rehman, Kashif Siddique \\ Department of Radiology, Shaukat Khanum Memorial Cancer Hospital and Research Centre, Lahore, \\ Pakistan
}

Received: 18 June 2021/Accepted: 09 July 2021

\section{Open ACCess Correspondence: \\ Afaque Ali, 7A Block R-3, Phase 2, M.A. Johar Town, Lahore - 54782, Punjab, Pakistan. Email: afaque. shaikh@hotmail.com \\ Citation: Ali A, Rehman S, Siddique K. Extraskeletal Mesenchymal Chondrosarcoma, a Rare Entity with Unusual Metastases: A Case Report. J Cancer Allied Spec [Internet]. 2021 May 31;7(2).e1002957. https://doi.org/10.37029/jcas. v7i2.411}

Copyright: () 2021 Ali, et al. This is an open access article distributed under the terms of the Creative Commons Attribution License, which permits unrestricted use, distribution, and reproduction in any medium, provided the original author and source are credited.

Funding: The authors received no financial support for the research, authorship and/or publication of this article.

Competing interest: Nil.

\begin{abstract}
Introduction: Extraskeletal mesenchymal chondrosarcoma (ESMC) is rare, aggressive, and high grade malignant tumors originating from soft tissues. It carries a poor prognosis with a tendency for local recurrence and distant metastasis, necessitating long-term follow-up. The most common sites for metastasis are the lungs, bones, and lymph nodes. Meanwhile, pancreatic metastases are extremely rare. Case Description: A 35-year-old female presented with a history of wide local excision for the left upper limb mass; histopathology showed ESMC. She was on surveillance with a computed tomography scan of the thorax and magnetic resonance imaging of the left upper limb at 3-months intervals until she developed vertebral and pancreatic lesions after 6 months post-surgery. No pulmonary metastases were noted. Considering the unusual site for metastasis and to exclude the possibility of any second malignancy, bone biopsy, and endoscopic ultrasound-guided fine-needle aspiration was performed that confirmed metastases. Later she developed osseous metastases in the pelvis and femora. Practical Implication: Pancreatic metastasis from ESMC is extremely rare. In case of new visceral or osseous lesions in a patient with a past medical history of ESMC, the possibility of metastatic disease should be considered. A biopsy can be performed to confirm the diagnosis.
\end{abstract}

Key words: Bones, cancer, extraskeletal mesenchymal chondrosarcoma, metastasis, pancreas

\section{Introduction}

Mesenchymal chondrosarcoma (MC) is a rare aggressive variant of chondrosarcoma, accounting for $<10 \%$ of chondrosarcomas. ${ }^{[1,2]}$ In contrast to conventional chondrosarcoma, only $1 \%$ of tumors are extraskeletal; $\mathrm{MC}$ has an extraskeletal location in $30-40 \%$ of cases. ${ }^{[3]}$ Extraskeletal MC (ESMC) represent about $1 \%$ of all chondrosarcomas. Based on their location two subtypes of ESMC have been described: tumors occurring in muscle and soft tissues and those associated with the central nervous system. ${ }^{[1,2]}$

ESMC differ from conventional chondrosarcoma in several aspects. Conventional chondrosarcomas show a male predilection and typically arise in the pelvis and femur. Whereas EMCs have a slight female preponderance and shows bimodal peak age incidences corresponding with predominant 
sites of origin. Central nervous system involvement occurs in patients aged $20-30$ years and soft tissue involvement occurs in 40 years and older. Compared to chondrosarcoma, MC are quite aggressive, and have 5 and 10 -year survival rates of $54.6 \%$ and $27.3 \%$, respectively. ${ }^{[3,4]}$

ESMC commonly involves the orbit, meninges, and lower limbs. Upper limb involvement is rare. ${ }^{[3]}$ Visceral involvement is also uncommon with few cases reported involving the kidney, spleen, pancreas and uterus. ${ }^{[5]}$ Tumor size ranges from 5 to $12 \mathrm{~cm}$ and are often well- circumscribed and covered with a thin fibrous capsule. ${ }^{[2]}$

ESMC carries a dismal prognosis because of rapid growth, and the tendency for local recurrence and distant metastasis. Therefore, these patients require long-term follow-up. ${ }^{[6]}$

We report the case of a 35-years old female with a diagnosis of ESMC involving left upper limb who later developed osseous and pancreatic metastases. Our hospital Institutional Review Board granted a waiver of informed consent to publish this case report.

\section{Case Description}

A 35-year-old female with a medical history of progressively increasing painless left arm swelling for 1 year, underwent excision biopsy in an outside hospital facility. Histopathology revealed that ESMC, $13.6 \mathrm{~cm}$ in size, was within $1 \mathrm{~mm}$ of the resection margin. Magnetic resonance imaging (MRI) left upper limb was performed that showed satisfactory post-surgical changes with no residual disease. Computed tomography (CT) scan through thorax was negative for pulmonary metastasis. Bone scan was also negative for distant osteoblastic metastasis. The case was discussed in Multidisciplinary Team (MDT), which recommended wide local excision of the scar. However, patient refused surgery and opted for surveillance.

Follow-up with CT thorax and MRI left upper limb was continued at three-month interval; there was no evidence of local disease recurrence or pulmonary metastasis. However, CT thorax at 6 months from presentation showed interval development of destructive lesion in T12 vertebral body with Grade 3 compression fracture and associated soft tissue component extending into spinal canal [Figure 1]. The patient was called to the emergency department, and a detailed medical history and physical examination were performed. She had a history of low back pain radiating to both legs for 2 months. However, there was no motor or sensory loss or sphincter dysfunction.

Further evaluation was done with MRI of the whole spine with contrast to exclude myelopathy. MRI redemonstrated Grade 3 pathological compression fracture of T12 vertebral body with suspicious marrow infiltration and extraosseous soft-tissue extending posteriorly within the spinal canal deep to the posterior longitudinal ligament causing effacement of the anterior thecal sac and compression of the conus medullaris. No edema seen within the conus medullaris [Figure 2]. The rest of the vertebral bodies were unremarkable with no evidence of metastasis. In addition to this, a note was made of a rim enhancing lesion in the body of the pancreas.

Furthermore, CT abdomen and pelvis with contrast was performed that showed a hypodense lesion measuring $13 \mathrm{~mm} \times 9 \mathrm{~mm}$ in the body of pancreas on

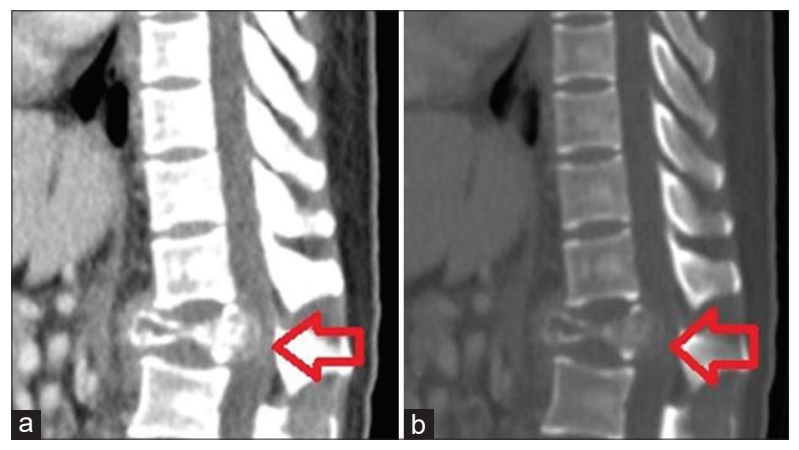

Figure 1: Computed tomography scan sagittal images (a) soft-tissue window and (b) bone window show destructive lesion in T12 vertebral body with Grade 3 compression fracture and extra osseous soft-tissue component extending into the spinal canal (red arrows) 
the background of normal pancreatic parenchyma without any surrounding fat arborization [Figure 3]. While the follow-up MRI of the left limb showed stable post-surgical changes with no evidence of local disease recurrence. These findings were discussed in MDT to decide the further management plan. As patient did not develop local recurrence and pulmonary metastasis; CT guided biopsy was recommended for T12 vertebral body lesion for histopathological confirmation of metastasis and exclude the possibility of second primary bone tumor. In addition, an endoscopic ultrasound (EUS) guided fine-needle aspiration
(FNA) for pancreatic lesion was planned. A CT guided bone biopsy was performed [Figure 4] and histopathology confirmed metastatic MC.

\section{Diagnosis and Management}

The patient was discussed in neurology MDT and was planned for spinal decompression and stabilization surgery. She underwent T10-L3 pedicle screw fixation, T12 Laminectomy and tumor debulking the by neurosurgery team. Histopathology of post-operative specimen again confirmed metastatic MC. After the patient
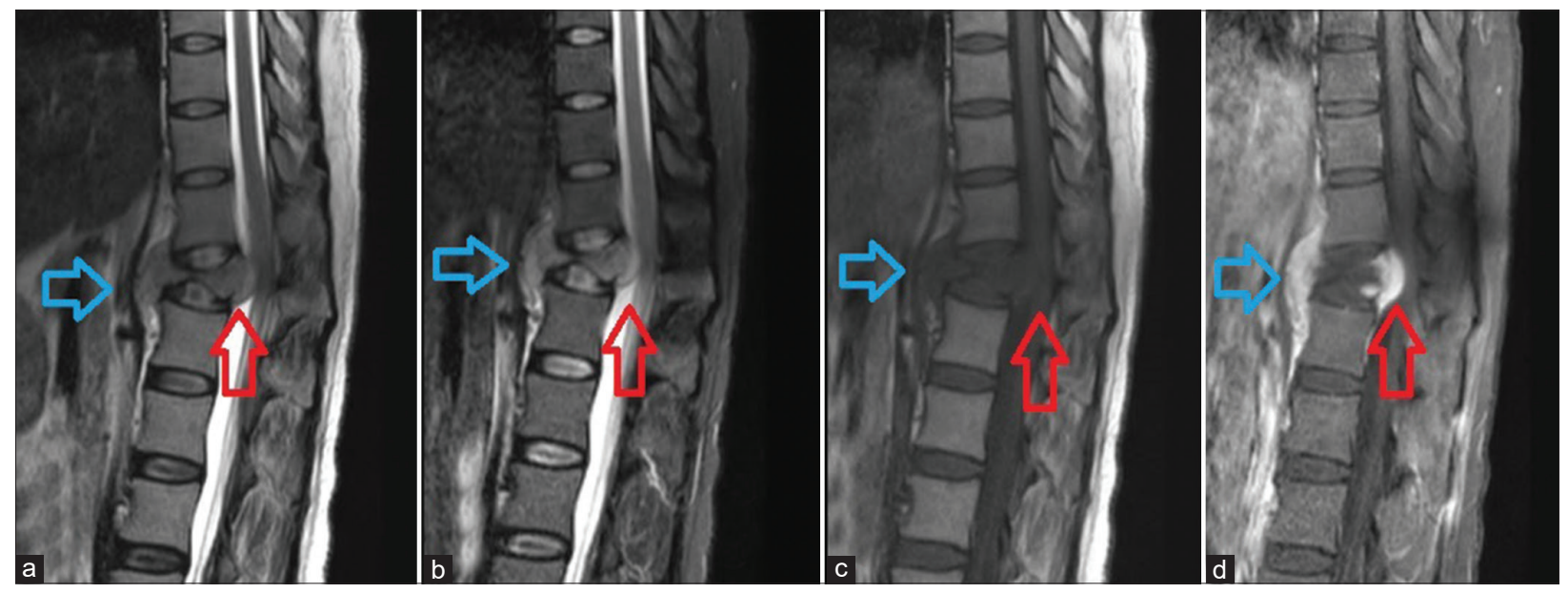

Figure 2: Sagittal magnetic resonance images through thoracolumbar spine show Grade 3 pathological compression fracture of T12 vertebral body with altered marrow signals; intermediate on T2-weighted imaging (WI) (a), hyperintense on Short Tau Inversion recovery sequence (b) and hypointense on T1WI (c). Post-contrast T1WI (d) shows enhancing soft tissue component extending posteriorly within spinal canal deep to posterior longitudinal ligament causing effacement of the anterior thecal sac and compression of the conus medullaris (red arrow). Enhancing soft-tissue component is noted anteriorly as well beneath anterior longitudinal ligament (blue arrow)
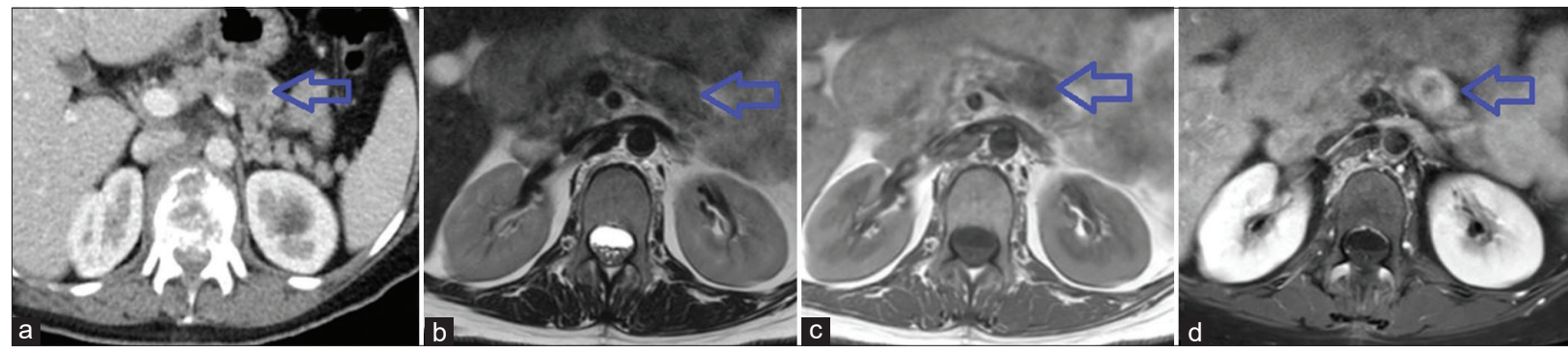

Figure 3: Contrast-enhanced computed tomography through abdomen axial section (a) shows a hypodense lesion in the body of the pancreas. This lesion was showing a heterogeneous signal on T2WI (b) and TIWI (c) and peripheral rim enhancement on post-contrast TIWI (d) in previously performed magnetic resonance imaging spine (shown by the blue arrow) 
had recovered from surgery, EUS guided FNA was performed for pancreatic lesion [Figure 5], cytopathology showed chondroid neoplasm, compatible with the patient's known history of MC.

She was started on Vincristine, Doxorubicin and Cyclophosphamide/Ifosfamide and Etoposide (VDC/IE) chemotherapy as per MDT discussion. However, while she was admitted for the fourth cycle of chemotherapy, she developed a worsening backache; without any apparent neurological deficit. MRI whole spine was performed that showed interval increase in extraosseous softtissue component and terminal spinal cord compression. Emergency radiotherapy was given to the thoracolumbar spine with a total dose of 2000c Gray in five fractions. Her pain was improved, and she was discharged on oral analgesics.

Follow-up CT showed marginal interval decrease in size of the pancreatic lesion, measuring $17 \mathrm{~mm}$ $\times 25 \mathrm{~mm}$ against the previous measurement of $22 \mathrm{~mm} \times 27 \mathrm{~mm}$ with the development of internal calcifications [Figure 6]. She underwent regular follow-up with CT chest abdomen and pelvis at intervals of 3 months. CT done after 6 months showed stable pancreatic lesion and changes of spinal fixation surgery; however, there was a development of lytic lesions in bilateral femora and left acetabulum [Figure 7]. Further evaluation

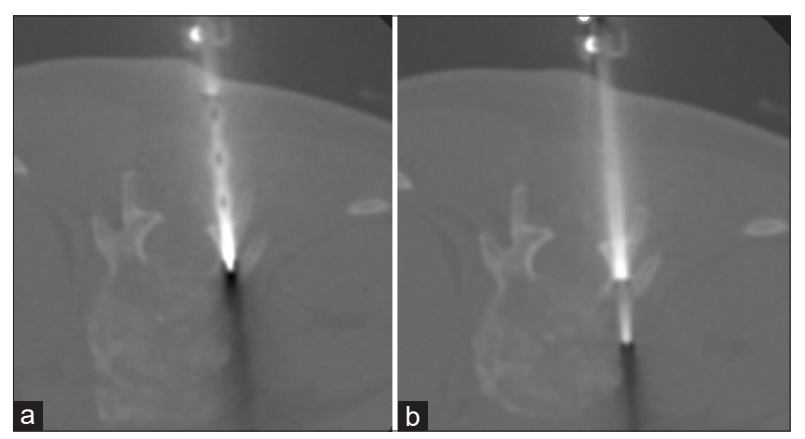

Figure 4: ( $a$ and b) show images of computed tomography-guided bone biopsy performed for T12 vertebral body lesion. A 10-G MADISON bone biopsy system was parked over the right pedicle of the T12 vertebra. Two cores were obtained from the T12 vertebral body using the transpedicle approach with MRI was performed that confirmed abnormal marrow signals consistent with metastasis [Figure 8]. The patient was evaluated by the orthopedic team. She had complaint of pain in both legs, which was more on the left side. She received prophylactic radiotherapy to the left femur (2000 cGray in 5 fractions), which resulted in pain improvement. Her examination and clinical review were unremarkable until the last follow-up. Additional follow-up imaging has not yet been done.

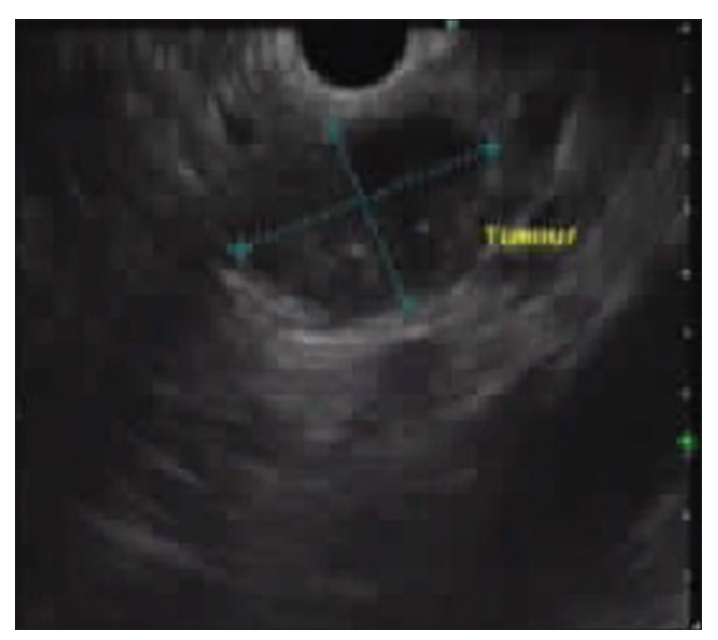

Figure 5: Endoscopic ultrasound image shows a $24 \mathrm{~mm}$ rounded solid, hypoechoic lesion in the body of the pancreas. Background pancreas tissue appeared normal. Using a 22 gauge needle, four passes were made and tissue obtained for cytologic evaluation.

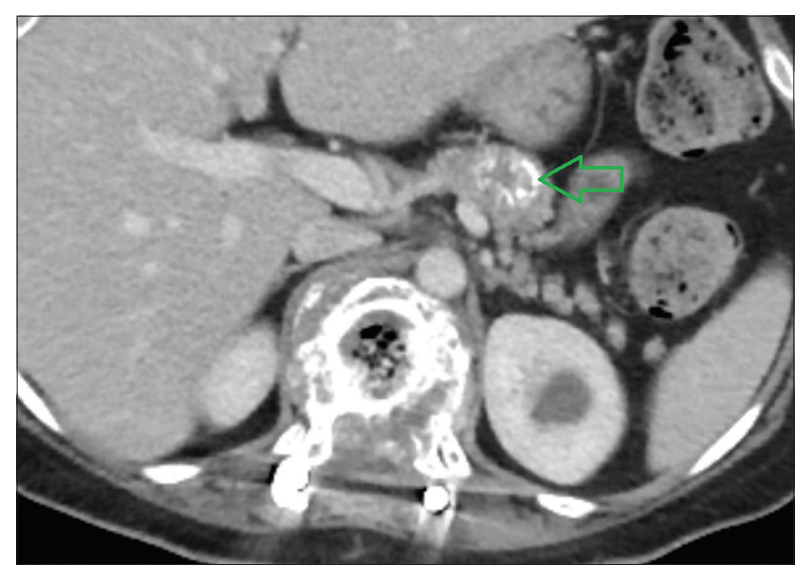

Figure 6: Axial contrast-enhanced computed tomography through abdomen shows the development of calcifications in metastatic pancreatic lesion 


\section{Discussion}

ESMC are rare high-grade malignant tumors originating from soft tissues. A plain radiograph is usually obtained as an initial investigation that often shows a soft tissue mass with areas of chondroid matrix appearing as arc-shaped or stippled calcification. However, there are limitations to this approach due to the overlapping of adjacent structures and poor resolution. ${ }^{[3,4]}$ On CT, ESMC appears as lobulated well-circumscribed softtissue mass with areas of granular, ring and arc or irregular coarse calcifications with peripheral postcontrast enhancement. ${ }^{[2,3]}$ On MRI, the soft-tissue component appears as isointense or hypointense on T1 weighted imaging ( $\mathrm{WI}$ ) and heterogenous on T2WI. Furthermore, mineralized and non-calcified areas appear as hypointense and hyperintense areas respectively. This is often termed as the black pepper sign. Post-contrast images show diffuse heterogeneous or nodular enhancement. ${ }^{[4]}$

Diagnosis is based on tumor morphology and various immunohistochemical stains. Histologically,

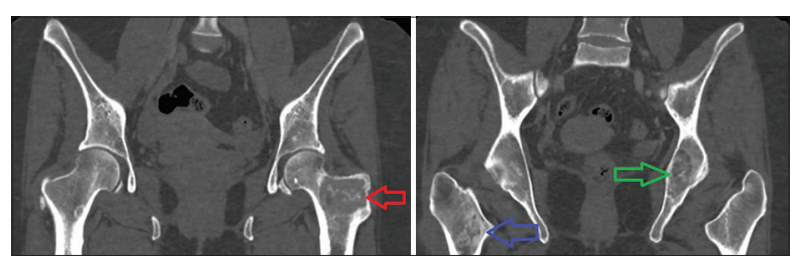

Figure 7: Computed tomography pelvis bone window coronal images ( $a$ and $b$ ) show lucent lesion with internal chondroid matrix involving the left femoral neck and greater trochanter (red arrow), right femur close to lesser trochanter (blue arrow), and left acetabulum (green arrow) the tumor has a bimorphic morphology composed of undifferentiated round blue cells and hyaline cartilage. ${ }^{[4,5]}$ EMCS can be challenging to diagnose with certainity in cases of small biopsy specimens that show only one of the two tissue elements especially in cases without cartilaginous components. The differential diagnosis for EMSC includes hemangiopericytoma, synovial sarcoma, malignant solitary fibrous tumor, Ewing Sarcoma, and other round blue cell tumors and other variants of chondrosarcoma. ${ }^{[2,3]}$

Because of the rarity of the tumor, the potential benefits of radiation and chemotherapy are unclear. Wide local excision of tumor is considered the main stay of treatment. According to the National Comprehensive Cancer Network guidelines recommendation, $\mathrm{MC}$ can be treated with VDC alternating with IE. This is a treatment regimen used typically in cases of Ewing's sarcoma. ${ }^{[4]}$

Metastasis from ESMC has been reported to occur in $<20 \%$ of patients occurring over a period of 10 months- 8 years. ${ }^{[6]}$ Pulmonary, bone, and lymph node metastasis are usually seen at the time of diagnosis. The most common site for metastasis are the lungs, and it carries a poor prognosis. ${ }^{[1]}$ The size of metastatic deposits ranges from $2 \mathrm{~cm}$ to $12 \mathrm{~cm} .{ }^{[5]}$

Osseous metastatic lesions appear as lytic lesions on plain radiographs. CT scans often demonstrate soft tissue density mass with calcification foci. ${ }^{[7]}$ These findings were also noted in our patient. Osseous metastatic lesions show increased uptake on bone scan and positron emission tomography/CT. ${ }^{[7,8]}$
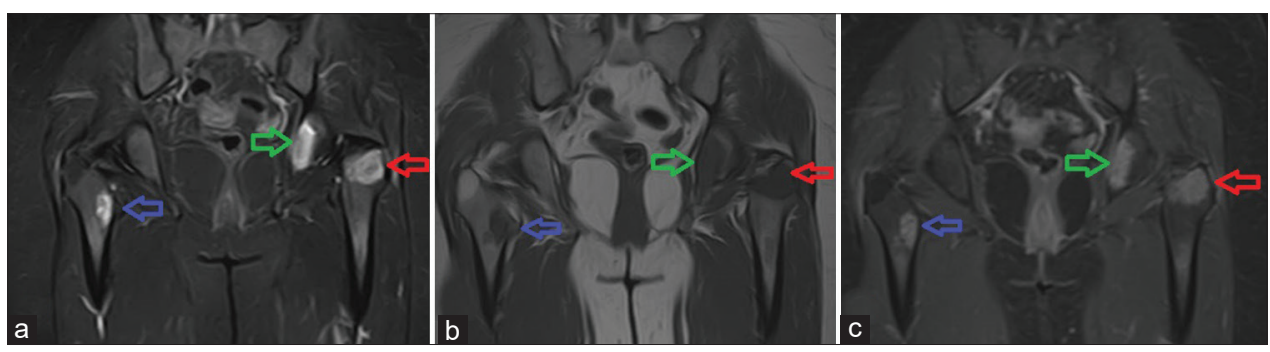

Figure 8: Magnetic resonance imaging pelvis coronal sections show STIR hyperintense (a), T1WI hypointense (b), enhancing lesions on post-contrast T1WI (c) in the right proximal femur near lesser trochanter (blue arrow), left acetabulum (green arrow), and left femoral neck and greater trochanter (red arrow) 
Although these imaging features suggest metastatic disease, bone biopsy helps in histopathological confirmation of diagnosis. ${ }^{[7,9]}$ Osseous metastatic lesions treated with radiotherapy often show calcification and well-defined sclerotic margin. ${ }^{[7]}$

Pancreatic metastases from ESMC are extremely rare. Only 8 cases have been previously reported in the literature with latency periods ranging from 3 years to 21 year and previously, just one case has been reported with synchronous presentation. $[5,8,10,11]$ Pancreatic metastases are usually accompanied by concurrent metastasis to other organs like lungs, lymph nodes, bones and kidneys. Primary ESMC involving the pancreas is also a rare entity with only two previously reported cases. ${ }^{[5]}$ Pancreatic metastatic lesions account for $<7 \%$ of all pancreatic masses, with the most common primary being kidney and lung. ${ }^{[11]}$ Patients can be asymptomatic and diagnosed on surveillance imaging. When symptomatic they usually present with abdominal pain and jaundice. ${ }^{[5]}$ On CT, metastatic pancreatic lesions appear as a hypodense tumor with granular irregular calcifications. On MRI tumor is hyperintense on $\mathrm{T} 2 \mathrm{WI}$ with internal low signal areas corresponding to calcifications. Our patient had an incidental diagnosis of pancreatic lesion, which demonstrated similar imaging features as detailed above. Calcifications were noted after treatment with an interval decrease in the size of tumor. The presence of a pancreatic lesion in a patient with a history of malignancy should raise suspicion of metastasis. EUS-guided fine-needle aspiration cytology should be performed to confirm the diagnosis. ${ }^{[5]}$

This case report is one of few cases of ESMC with several unique features. Firstly, ESMC involving muscle and soft tissue most commonly arises from the lower limb. Upper limb involvement has an extremely rare occurrence. Second, lungs are the most common site for metastatic disease, which was not seen in our case; instead the patient developed pancreatic metastasis, which is exceedingly rare. Imaging finds its role in longterm follow-up of these patients to detect the development of metastasis and local recurrence, thereby improving long term survival.

\section{Acknowledgments}

None.

\section{References}

1. Ghaffari S, Farsavian A, Daneshpoor SM, Azar MS. Extraskeletal mesenchymal chondrosarcoma of shoulder: An extremely rare case. J Orthop Case Rep 2016;6:35-8.

2. Arora K, Riddle ND. Extraskeletal mesenchymal chondrosarcoma. Arch Pathol Lab Med 2018;142:1421-4.

3. Seo CY, Jung ST, Byun JW. Extraskeletal mesenchymal chondrosarcoma in the axillary region: Reports of two cases. Korean J Pathol 2012;46:483-8.

4. Hunter K, Alexander A, Passerini S, Rovner A, Garg A. Extraskeletal mesenchymal chondrosarcoma arising in adductor magnus with metastatic foci. BJR Case Rep 2015;2:20150117.

5. Shah SN, Parameswaran A, Reddy PK. Metastatic extraskeletal mesenchymal chondrosarcoma of the pancreas: Report of an unusual case with review of literature. Asian J Oncol 2021:1-4.

6. Tulic G, Nagulic M, Sopta J, Todorovic A. Metastatic or multicentric? A report of what we believe to be a case of multicentric extraskeletal mesenchymal chondrosarcoma. Eur J Orthop Surg Traumatol 2007; 17:173-7.

7. Malhotra CM, Doolittle CH, Rodil JV, Vezeridis MP. Mesenchymal chondrosarcoma of the kidney. Cancer 1984;54:2495-9.

8. Tsukamoto S, Honoki K, Kido A, Fujii H, Enomoto Y, Ohbayashi $\mathrm{C}$, et al. Chemotherapy improved prognosis of mesenchymal chondrosarcoma with rare metastasis to the pancreas. Case Rep Oncol Med 2014;2014:249757.

9. Zhang $M$, Zhang H, Xia L, Qu Y, Wang H, Yu C. A case of tentorial mesenchymal chondrosarcoma with bone metastases. Neurosurg Q 2013;23:148-52.

10. Yamamoto $H$, Watanabe $K$, Nagata $M$, Honda I, Watanabe $\mathrm{S}$, Soda $\mathrm{H}$, et al. Surgical treatment for pancreatic metastasis from soft-tissue sarcoma: Report of two cases. Am J Clin Oncol 2001;24:198-200.

11. Smith AL, Odronic SI, Springer BS, Reynolds JP. Solid tumor metastases to the pancreas diagnosed by FNA: A single-institution experience and review of the literature. Cancer Cytopathol 2015;123:347-55.

\section{Authorship Contributions}

Conceived and designed the analysis: AA, KS. Collected the data: AA. Contributed data or analysis tools: SR Performed the analysis: AA, SR Wrote the paper: AA, SR, KS. 